\title{
COVID-19 Vaccine Failure in Chronic Lymphocytic Leukemia and Monoclonal B- Lymphocytosis; Humoral and Cellular Immunity
}

Yandong Shen ${ }^{1,2}$, Jane A. Freeman ${ }^{3,4}$, Juliette Holland ${ }^{5}$, Ann Solterbeck ${ }^{6}$, Kartik Naidu ${ }^{5}$, Asha Soosapilla ${ }^{3}$, Paul Downe ${ }^{3}$, Catherine Tang $^{3}$, lan Kerridge ${ }^{1}$, Lucinda Wallman ${ }^{7}$, Nenna Van Bilsen $^{3}$, Vanessa Milogiannakis ${ }^{8}$, Anouschka Akerman ${ }^{8}$, Gabriela Martins Costa Gomes ${ }^{9}$, Kerrie Sandgren ${ }^{9}$, Anthony L Cunningham ${ }^{9}$, Stuart Turville ${ }^{8}$, Stephen P. Mulligan ${ }^{1,2,3}$

1. Department of Haematology, Royal North Shore Hospital, St Leonards, NSW Australia

2. Kolling Institute, Royal North Shore Hospital, St Leonards, NSW Australia

3. Department of Haematology and Flow Cytometry, Laverty Pathology, Macquarie Park NSW Australia

4. Northern Haematology and Oncology Group, Sydney Adventist Hospital, Wahroonga NSW Australia

5. Department of Microbiology, Laverty Pathology, Macquarie Park, NSW Australia

6. Statistical Revelations Pty Ltd, 41 The Parade, Ocean Grove, VIC Australia

7. Department of Immunology, Laverty Pathology, Macquarie Park, NSW Australia.

8. Kirby Institute, University of New South Wales, Kensington, NSW Australia

9. Centre for Virology Research, Westmead Institute, Sydney Infectious Diseases University of Sydney, NSW Australia

*Corresponding authors:

Dr. Yandong Shen and Prof Stephen Mulligan

Department of Hematology, Kolling Institute of Medical Research, Royal North Shore Hospital, Sydney NSW 2065 Australia

E-mails:

1. yandong.shen@sydney.edu.au

2. mulligan@sydney.edu.au

$\begin{array}{ll}\text { Abstract } & 250 \text { words } \\ \text { Main text } & 3957 \text { words } \\ \text { References } & 43 \\ \text { Tables - main } & 4 \\ \text { Figures - main } & 3 \\ \text { Supplementary tables } & 5 \\ \text { Supplementary figures } & 1\end{array}$




\section{Key Novel Findings}

1. Comparison CLL vs MBL vs normal

- $45 \%$ of CLL and $9.5 \%$ of MBL fail to seroconvert with 2 doses of COVID-19 vaccine

2. Neutralization assay

- SARS CoV-2 IgG levels $<1000 \mathrm{AU} / \mathrm{mL}$ rarely associated with neutralization activity.

3. COVID-19-specific T-cell function by FluoroSpot IFN-g and IL-2 production

4. IgG, A, M class and IgG subclass: correlations by univariate and multivariate analysis

- IgM (OR 7.29 p<0.0001), IgG2 and IgG3 subclass univariate significance

5. Correlation with therapy - ICT, targeted therapies, and those on Ig replacement

6. High risk of vaccination failure for all CLL, including early-stage disease, and MBL

\section{Key Points}

CLL and MBL show significantly impaired anti-spike antibody, viral neutralization, with cellular immune response to COVID-19 vaccination

Failure to seroconvert is associated with low $\operatorname{lgM}, \operatorname{lgG} 2, \operatorname{lgG} 3$, and recent therapy; many CLL and MBL patients remain COVID-19 vulnerable 


\section{Abstract}

Chronic lymphocytic leukemia (CLL) is associated with immunocompromise and high risk of severe COVID-19 disease and mortality. Monoclonal B-Lymphocytosis (MBL) patients also have immune impairment. We evaluated humoral and cellular immune responses in 181 patients with CLL (160) and MBL (21) to correlate failed seroconversion (<50AU/mL SARSCoV-2 II IgG assay, antibody to spike protein, Abbott Diagnostics) following each of 2 vaccine doses with clinical and laboratory parameters. Following first and second doses, $79.2 \%$ then $45 \%$ of $\mathrm{CLL}$, and $50 \%$ then $9.5 \%$ of $\mathrm{MBL}$ respectively remained seronegative, indicating 2 vaccine doses are crucial. There was significant association between post-dose 2 antibody level with pre-vaccination reduced $\operatorname{IgM}(p<0.0001), \lg G 2(p<0.035), \lg \mathrm{g} 3(p<0.046)$, and CLL therapy within 12 months $(p<0.001)$ in univariate analysis. By multivariate analysis, reduced $\lg M(p<0.0002)$ and active therapy $(p<0.0002)$ retained significance. There was no significant correlation with age, gender, CLL duration, IgG, IgA or lymphocyte subsets. Anti-spike protein levels varied widely and were lower in CLL, than MBL, and both lower than normal donors. Neutralization activity showed anti-spike levels $<1000 \mathrm{AU} / \mathrm{mL}$ were usually negative for both an early viral clade and the contemporary Delta variant. There were $72.9 \%$ of CLL and $53.3 \%$ of $\mathrm{MBL}$ who failed to reach anti-spike levels $>1000 \mathrm{AU} / \mathrm{mL}$. In a representative subset of 32 CLL patients, $80 \%$ had normal T-cell responses by IFN $\gamma$ and IL-2 FluoroSpot assay. Failed seroconversion occurred in $36.6 \% \%$ of treatment-naive patients, $52.9 \%$ treatment-naive with reduced IgM, $78.1 \%$ on therapy, and $85.7 \%$ on ibrutinib. Vaccination failure is very common in CLL, including early-stage disease. 


\section{Introduction}

Chronic Lymphocytic Leukemia (CLL) is almost invariably associated with some degree of immune failure, that typically worsens over the course of the disease. ${ }^{1-3}$ About $85 \%$ of CLL patients ultimately develop hypogammaglobulinemia, but also a wide range of both humoral and cell-mediated immune defects. ${ }^{2,3}$ This predisposes to higher risk of infection ${ }^{4,5}$ and second malignancy. ${ }^{6-8}$ There is a higher frequency and severity of viral diseases such as herpes zoster, ${ }^{9,10}$ and for some, such as rhinovirus, ${ }^{11,12}$ there is impaired ability to clear the virus.

Severe Acute Respiratory Syndrome Corona-virus-type 2 (SARS-CoV-2) is a single-stranded RNA virus that causes human infection, and COVID-19 disease manifestations, range from asymptomatic through to severe respiratory failure and death. ${ }^{13}$ COVID-19 has a major impact in patients with CLL. ${ }^{14,15}$ Data from the European Research Initiative on CLL (ERIC) show in CLL patients with confirmed COVID-19, 79\% developed severe disease and require hospitalization and $36.4 \%$ died. ${ }^{16}$ Even in recent waves of the disease, mortality rates of $11 \%$ have been typical. ${ }^{17}$ Prolonged live virus shedding of SARS-CoV-2 has also been documented in $\mathrm{CLL}^{18,19}$

Since the advent of COVID-19 vaccines, CLL patients have been included in national and global vaccination programs. Early data indicate that failure of seroconversion and probable ongoing COVID-19 susceptibility is a problem in CLL. ${ }^{20-22}$ For many, SARS-CoV-2 infection is likely resulting in major immediate risk of morbidity and mortality. We sought to better understand the immune impairments that predict failure of seroconversion by analyzing $B$ and T-cell immune responses to COVID-19 vaccines and correlating with a wide range of 
clinical, therapeutic, and biological characteristics including quantification of immunoglobulins (G, A, M, IgG subclasses), lymphocyte subsets, and COVID-19-specific neutralization antibody and T-cell responses.

\section{Methods}

The study was approved by the Northern Sydney Local Health District Human Research Ethics Committee (approval number: LNR/14/HAWKE/181) and all patients provided an informed consent. The diagnosis of CLL and MBL were according to iwCLL guidelines. ${ }^{23}$ Vaccination occurred through the Australian Government vaccination program (www.health.gov.au) where the main initial vaccine availability was internationally and domestically produced Vaxzevria (AstraZeneca AZ), and more recently, Comirnaty (Pfizer) and Spikevax (Moderna) mRNA vaccines. ${ }^{24}$ Blood samples (FBC, biochemistry, immunoglobulins G, A, M, IgG subclasses, phenotyping and COVID-19 antibody levels) were taken pre-vaccination, then following vaccination doses 1 (D1) and 2 (D2) approximately 2 to 4 weeks following each dose. CLL therapy was avoided unless essential or given in the periods when there was no community COVID-19 transmission. ${ }^{25}$

$\operatorname{lgG}(6.5-16 \mathrm{~g} / \mathrm{L}), \mathrm{A}(0.4-3.5 \mathrm{~g} / \mathrm{L})$ and $\mathrm{M}(0.53-3 \mathrm{~g} / \mathrm{L})$ were performed on the Siemens Atellica. IgG subclasses (IgG1, 3.82-9.29g/L; IgG2, 2.42-7g/L; IgG3, 0.22-1.76g/L; IgG4, 0.04-0.86g/L) were performed using Optilite IgG subclass kits (The Binding Site). Analysis of IgG and IgG subclass data excluded all patients on immunoglobin replacement therapy (IgRT). Flow 
cytometry was performed on a Navios 10 color instrument with Beckman-Coulter fluorochrome-labelled monoclonal antibodies.

SARS-CoV-2 IgG II Quant assay ${ }^{\circledR}$ (Abbott Diagnostics) was performed in accordance with the manufacturer's instructions. This chemiluminescent microparticle immunoassay detects IgG antibodies to the spike receptor-binding domain (RBD) of the S1 subunit of the spike protein of SARS-CoV-2. The SARS-CoV-2 antigen-coated paramagnetic microparticles bind to the IgG antibodies against the virus' spike protein in human serum and plasma samples. The resulting chemiluminescence in relative light units (RLU) following the addition of acridinium labeled anti-human IgG (mouse, monoclonal) in comparison with the IgG II calibrator/standard indicates the strength of response, which reflects the quantity of RBD IgG present. Fifty arbitrary units per milliliter $(50 \mathrm{AU} / \mathrm{mL})$ and above in this test are considered positive. For this study, we also examined the degree of anti-spike protein response with arbitrary strata of $<50$ (negative), 50-249 (weak), 250-999 (moderate), 10004999 (strong), 5000-9999 (high), and >10000 (very high).

\section{SARS-CoV-2 live virus neutralization assay:}

HEK-ACE2/TMPRSS cells (Clone 24$)^{26}$ were seeded in 384 -well plates at $5 \times 10^{\wedge} 3$ cells per well in the presence of the live cell nuclear stain Hoechst-33342 dye (NucBlue, Invitrogen) at a concentration of $5 \% \mathrm{v} / \mathrm{v}$. Two-fold dilutions of patient plasma samples were mixed with an equal volume of SARS-CoV-2 virus solution $\left(1.25 \times 10^{\wedge} 4 \mathrm{TCID} 50 / \mathrm{mL}\right)$ and incubated at $37^{\circ} \mathrm{C}$ for 1 hour before adding $40 \mu \mathrm{l}$ in duplicate to the cells (final $\mathrm{MOI}=0.05$ ). Viral variants used included the variants of concern; Delta (B.1.617.2), as well as 'wild-type' control virus (B.1.319/D614G strain) from an early circuiting 2020 clade (B.1). Plates were incubated for 
24 hours post infection and entire wells were imaged by high-content fluorescence microscopy, cell counts obtained with automated image analysis software, and the percentage of virus neutralization was calculated with the formula: $\% \mathrm{~N}=(D-(1-Q)) \times 100 / D$,

as previously described. ${ }^{26}$ An average $\% \mathrm{~N}>50 \%$ was defined as having neutralizing activity. In studies of convalescent patients, $96 \%$ of COVID-19 infected, convalescent patients reach a titer of $1 / 40$ at their peak and is therefore used as the benchmark titer in this study. ${ }^{26}$ The mean titer of the WHO G serology standard in this assay for the above B1 clade virus is $1 / 637$.

\section{IFNg/IL-2 Fluorospot Assay}

PBMCs isolated from whole blood by Ficoll-Hypaque density gradient centrifugation were seeded in T-cell interferon gamma (IFNY)/interleukin-2 (IL-2) dual color fluorospot plates (Mabtech). For patients with a CD3\% within the normal reference interval, 250,000 cells per well were plated. For patients outside the reference interval, the cell number was normalized according to the patient's CD3\%, derived by flow cytometry on whole blood, to give either 100,000 (for low CD3\%) or 220,000 (for high CD3\%) T cells in a maximum of 400,000 cells per well. Where a patient's CD3\% was less than $20 \%$, the maximum 400,000 cells per well was plated. Cells were incubated with an overlapping peptide pool spanning the complete S protein $(2 \mu \mathrm{g} / \mathrm{mL}$; Miltenyi) for $18 \mathrm{~h}$ at $37 \mathrm{C}$. Negative control wells lacked peptides and PHA (10 $\mathrm{\mu g} / \mathrm{mL}$; Vector Laboratories) was used as a positive control. Plates were developed according to the manufacturer's instructions and read on a Mabtech Iris fluorospot reader. All tests were performed in duplicate, and the mean value was used for data representation. 


\section{Statistical analyses}

All analyses were conducted using SAS V9.4. The proportion of patients in various categories for response to COVID-19 vaccine and clinical variables are presented with exact 95\% confidence limits. The association between COVID-19 vaccine response (negative/positive) after the second dose of vaccine and clinical variables was estimated using univariate logistic regression models, modelling the odds of a negative response, fitted with conditional exact methods to obtain exact P-values and 95\% confidence limits. Exact methods were employed due to the small patient numbers in some categories. Multivariate logistic regression models, modelling the odds of a negative response after the second dose of vaccine, were fitted including multiple clinical variables found to be significant in univariate models. If two clinical variables were highly correlated (e.g. treatment within last 12 months and currently on treatment) only one variable was included. In addition, a final model was fitted including only terms statistically significant in the more inclusive multivariate model. Note, any analyses where pre-vaccination IgG levels (or IgG subclasses) were considered, patients currently treated with IgG replacement therapy were excluded.

\section{Results}

\section{Patient characteristics}

From $1^{\text {st }}$ March through to $22^{\text {nd }}$ October 2021 , a total of 235 patients, 206 CLL and $29 \mathrm{MBL}$, were assessed. Statistical analysis only included patients with post D2 vaccine response available, i.e., $160 \mathrm{CLL}$ and $21 \mathrm{MBL}$, in total 181 patients. The patient baseline demographic 
characteristics are shown in Table 1 . The median age was 71.5 years for CLL, and 71 years for MBL. The proportion of males for CLL was $56.3 \%$, and for MBL was $38.1 \%$. The time from CLL diagnosis to vaccination ranged from $<1$ to 32 years with a median of 10 years.

There were 82 patients with CLL who were treatment-naïve, 40 previously treated in complete or partial remission, 6 with progressive disease pending therapy, and 32 on active therapy. Of the later there were 21 on ibrutinib, 2 on FCR (fludarabine, cyclophosphamide, rituximab), 1 bendamustine plus rituximab, 4 venetoclax ( 1 venetoclax/ibrutinib), and 4 on other therapies. The majority (both CLL and $M B L) 149$ received $A Z$ while 23 received Pfizer, and 1 received Moderna for both D1 \& D2, while for 8 patients, the type of vaccination was unclear. No difference in anti-spike antibody efficacy was identified with the different vaccines. No patients had COVID-19 infection prior to vaccination. There were no thromboembolic events cerebral or otherwise with the $A Z$ vaccine, and no episodes of pericarditis or myocarditis reported with Pfizer.

\section{Antibody responses}

Antibody responses to COVID-19 spike protein (anti-S antibody) using the SARS-CoV-2 II IgG assay Abbott Diagnostics assay as qualitatively negative or positive (i.e. IgG level $<50 \mathrm{AU} / \mathrm{mL}$ or $\geq 50 \mathrm{AU} / \mathrm{mL}$ ) after D1 and D2 respectively are shown in Table 2. In CLL patients ( $=160$ ), 79.2\% were negative after D1 and $45.0 \%$ remained negative after D2. For the $21 \mathrm{MBL}$ patients, $40.0 \%$ and $9.5 \%$, respectively were negative after each dose, compared to $0 \%$ and $0 \%$ of 25 normal controls (i.e. $100 \%$ of 25 normal controls seroconverted after D1) (Table 3 and Figure $1 \mathrm{~A}$ ). No patient or control had an anti-nucleocapsid antibody response prior to vaccination, consistent with absence of COVID-19 infection (data not shown). 


\section{Correlations with anti-S protein serologic response}

Statistically significant associations were identified between failure to achieve an anti-S response with pre-vaccination low IgM (odds ratio [OR] 7.29, $p<0.0001$ ), low IgG2 (OR 2.52; $p=0.035$ ), low IgG3 (OR 2.68; $p=0.046$ ) and therapy within the prior 12 months (OR 5.20; $p<0.0001$ ) (Figure 2 and Supplementary Table 1). We did not find statistically significant associations with age, sex, or duration of CLL. Neither IgA (OR 1.69) or total IgG (OR 1.84; $p=0.16$ ) reached statistical significance. All analysis of $\operatorname{Ig} G$ and $\operatorname{Ig} G$ subclasses excluded patients on IgRT. There was no correlation with the CLL or MBL clonal population level, nonclonal B-cell numbers, total CD3+, nor CD4+ or CD8+ T-cells, and nor with memory CD4+ or memory CD8+ T-cell populations (data not shown). There was no correlation with CLL cytogenetics or immunoglobulin heavy chain variable region genes (IGHV) mutational status.

Therapy in the prior 12 months was strongly associated with poor antibody response, with 74.4\% failing to seroconvert (OR 5.20; $\mathrm{p}<0.0001$ ) (Figure $2 \mathrm{~A}$ and Supplementary Table 2). Some patients $(n=6)$ with disease progression had their therapy deferred to minimize COVID-19 risk. For patients on active CLL-directed therapy, $78.1 \%$ remained seronegative after 2 vaccine doses (Supplementary Table 2). For those on active Btk-inhibition, mostly ibrutinib, 18 out of 21 failed to seroconvert (85.7\%), and for those treated in the last 12 months, so did 7 of 9 receiving anti-CD20 (77.8\%), 3 of 4 FCR, and 3 of 4 venetoclax. Interestingly, 2 of 2 patients on low dose prednisone ( $5 \mathrm{mg}$ daily) only for autoimmune cytopenia, and never on CLL-directed therapy had no seroconversion. Of CLL patients on IgRT for hypogammaglobulinemia with recurrent or severe infections, $77.3 \%$ (OR 5.18; $p=0.002$ ) failed to seroconvert (Figure 2A and Supplementary Table 2). 
There were $82(51.3 \%)$ treatment-naive patients 30 (36.6\%) of whom failed to seroconvert. $52.9 \%$ of patients with reduced IgM as the only immunoglobulin class reduction failed to seroconvert.

\section{Multivariate analysis of serologic response in patients with CLL}

In a multivariate analysis, we evaluated parameters associated with failure to seroconvert, namely pre-vaccination $\lg G 2$ and $\lg G 3$ levels (not on $\operatorname{IgRT}$ ), IgM levels and on-going treatment. Pre-vaccination IgM levels (OR 9.31; $p=0.0002$ ) and on-going therapy (OR 13.09; $p=0.0002$ ) remained predictors of negative serologic response. Pre-vaccination IgG2 (OR 1.19; $p=0.69$ ) and $\operatorname{lgG}(\mathrm{OR} 2.11 ; p=0.13$ ) lost significance in multivariate analysis.

\section{Variation in anti-spike protein titers in CLL, MBL and normal}

Anti-S levels in CLL demonstrated both a high proportion of negative results, and low levels compared to healthy controls (Figure 1). There was a wide range of anti-S levels from zero to $>40,000$. We divided these into six strata from negative to very high (Methods and Table 3) and measured neutralizing antibody in each stratum.

\section{Neutralization assay and anti-spike antibody levels}

Neutralizing capacity of 30 patients ( 5 patients from each stratum) measured against both the original D614G and Delta SARS-CoV-2 variants are shown in Figure $3 \mathrm{~A}$ and Supplementary Table 4. Of the 30 patients, neutralizing activity against the D614G strain was present in 12 (40\%); 11 of those 12 had anti-S levels $>1000$, while 1 was $95 \mathrm{AU} / \mathrm{mL}$. Of the 30 patients, neutralizing activity against the Delta variant was seen in 9 (30\%) and 8 had 
Anti-S levels $>1000$, and 1 was $875 \mathrm{AU} / \mathrm{mL}$. Hence, the majority of patients with neutralizing antibody had an anti-S level of $>1000$. Only 3 of 18 patients with anti-S level $<1000$ had detectable neutralizing activity.

In view of the almost invariable absence of neutralizing activity with anti-S level $<1000 \mathrm{AU} / \mathrm{mL}$, we evaluated the parameters associated with failure to achieve an anti-S level of $>1000$. The data are shown in Supplementary Tables 1 and 3. After D2, 73.0\% of CLL and $53.3 \%$ of $\mathrm{MBL}$ failed to reach an anti-S level $>1000$. Statistically significant associations between an anti-S level <1000, and IgM, IgG3 and treatment within the last 12 months remained, and IgA became statistically significant. The OR for total IgG was $2.75(p=0.060)$, for $\operatorname{lgM} 2.81(p=0.024)$, for $\lg A 3.30(p=0.043)$, and for $\lg 333.84(p=0.046)$. For patients on active therapy, $90 \%$ failed to achieve anti-S level $>1000$ (OR on therapy vs naive/prior therapy $4.27, p=0.0659)$.

\section{Cell-mediated and COVID-19 specific T cell response}

COVID-19 specific IFNy and IL-2 responses were measured in 31 patients by the FluoroSpot assay across a range of anti-S antibody and clinical settings, many from the cohort with neutralizing assay results (Figure 3B). Overall, COVID-19 peptide stimulated T-cells from freshly collected blood samples showed essentially normal function in most patients. There was no correlation with either anti-S antibody production, nor with neutralizing activity. Of the 31 patients, 25 (80\%) had a normal response, albeit of variable intensity, while 6 (20\%) had a weak or negative response. Good T-cell function was present in several patients with prior and current therapy. There was no clear correlation between T-cell function and any clinical or therapeutic parameter. 


\section{Discussion}

CLL patients are virtually all immunocompromised to some extent ${ }^{1,2}$ and while SARS-CoV-2 vaccines have proven successful in protecting the general community, early data suggest that many patients with CLL fail vaccination ${ }^{20-22}$. In this study we examined patients across the spectrum of CLL, from those with a small peripheral blood clone, $\mathrm{MBL}^{27,28}$, to early stage CLL through to advanced stage and heavily treated CLL. Patients who fail to mount an antibody response likely remain COVID-19 susceptible and vulnerable to severe COVID-19 disease, hospitalization, intensive care unit (ICU) admission, and death. ${ }^{15}$ We therefore focused on this vulnerable group who failed to seroconvert examining both the humoral and cell-mediated immune response.

The study demonstrates a high proportion of CLL patients fail to achieve a positive anti-S level greater than the assay threshold of $50 \mathrm{AU} / \mathrm{mL}$, with $79.2 \%$ and $45 \%$ failing seroconversion after $\mathrm{D} 1$ and $\mathrm{D} 2$, respectively (Table 2). In MBL, 50\% and $9.5 \%$ remained seronegative after D1 and D2. By contrast, a group of normal controls all achieved a positive anti-S level after D1, with much higher levels after D2. This emphasizes the importance of 2 vaccine doses in patients with CLL, and MBL. All patients with MBL by definition, and a high proportion of early stage CLL have few or no clinical problems with their disease, and many have minimal if any infection risk prior to COVID-19. Many of these patients do not mount a COVID-19 vaccine antibody response. In view of the indolent nature of their CLL, COVID-19 is now likely their highest risk of morbidity and mortality, far exceeding that from the CLL (or $M B L)$ itself. 
Parameters associated with failure to achieve a positive anti-S level in univariate analysis were pre-vaccination reduced $\lg M, \lg 22, \lg G 3$, and $C L L$ therapy within 12 months ( $p$ $<0.0001,0.035,0.046$ and $<0.001$ respectively). There was no significant correlation with age, gender, CLL duration, IgG, IgA or lymphocyte subsets. Patients aged 65 years and older had a potentially meaningful higher risk of vaccine failure with an OR of 1.34 , overall similar to data reported by Parry et al. ${ }^{20}$ and Herishanu et al..$^{21}$.

Evaluating total IgG only in patients not on IgRT, the OR for pre-vaccination IgG was 1.84 but did not reach significance ( $p=0.17$ ). There was a novel correlation with IgG2 and IgG3 and failure to seroconvert, but not $\lg G 1$, raising the interesting hypothesis that $\lg G 2$ and $\lg G 3$ may be more important than IgG1 for COVID-19 vaccine response. ${ }^{29}$ Furthermore, the lack of difference with response and IgG1 may contribute to why total IgG did not reach significance. Unlike 2 other studies ${ }^{20,21}$, we did not find significance with IgA, but this did emerge as significant when considering anti-S level $>1000 \mathrm{AU} / \mathrm{mL}$ as a strong serologic response.

Clinical variables significant in univariate analyses were considered in a multivariate model. Reduced IgM, prior treatment in the last 12 months, currently on treatment, current/prior IgG replacement therapy, reduced IgG2 and reduced IgG3 were all statistically significant at the $p<0.05$ level by univariate analysis. Due to the high correlation between any treatment in the last 12 months and currently on treatment, only "currently on treatment" was included in the model and enabled direct comparison with Israeli data ${ }^{21}$. Consistent with Herishanu et $a .^{21}$, currently on treatment (OR 13.09; $p=0.0002$ ) and IgM (OR 9.31; $p=0.0002$ ), but not $\lg A$, were predictors of serologic responses to vaccines. We did not find association between total IgG and vaccine responses. The OR for pre-vaccination $\lg G 3$ was 
2.11 did not reach significance $(p=0.13)$. Low $\lg G 2$ and low $\operatorname{lgM}$ were highly correlated as $45 \%$ of CLL patients had both (Supplementary data Table 5).

The strong association of failure to respond to vaccination with a low IgM level, often as a sole immunoglobulin class abnormality, is of concern as this is present in over a half of all CLL patients at diagnosis and in early-stage disease. ${ }^{2}$ This highlights the large numbers of CLL patients at ongoing risk, but also the importance of IgM and the primary immune response in developing antibodies.

Regarding CLL patients on therapy, as also demonstrated by others, there was a very strong association with vaccination failure in $74.4 \%$ (OR $5.20, p<0.001)$ of patients treated within the last 12 months, and $78 \%$ of those currently on active therapy failing to respond. Indeed, we found markedly impaired vaccine response with all forms of therapy with 18/21 ibrutinib, $^{30} 7 / 9 \mathrm{CD} 20$ antibody, ${ }^{31} 3 / 4$ FCR and $3 / 4$ venetoclax all failing to seroconvert.

Patients requiring IgRT as expected had very low vaccine response with $77.3 \%$ (OR 5.18; $p=0.002)$ failing to develop antibodies. In Australia, the criteria for IgRT access for CLL patients are the presence of both hypogammaglobulinemia, and "recurrent or severe infection". Hence this group is a surrogate for the "history of severe infection" group reported by Parry et al. ${ }^{20}$ and with a highly comparable response and failure rate.

Anti-S levels varied widely with many low, and of note lower in CLL, than in MBL, and in turn both were lower than healthy donors. Neutralization assays performed across the wide range of anti-S levels showed that neutralization activity was almost entirely restricted to those with an anti-S level $>1000 \mathrm{AU} / \mathrm{mL}$. Using $1000 \mathrm{AU} / \mathrm{mL}$ as the threshold of response, 
73.0\% of CLL and $53.3 \%$ of MBL failed to achieve this level. Also of note was that viral neutralization titers appeared weaker for the now globally dominant Delta variant compared to the original D614G strain. This is not unexpected as the vaccines were designed for D614G, but it is reassuring that significant activity against the Delta variant was demonstrated. T-cell responses appeared relatively intact, including those having received prior FCR and those on active therapy. ${ }^{32}$ Some patients appeared to have adequate T-cell numbers but failed to respond. Similarly, we found no significant correlation with total CD3+, nor CD4+ or CD8+ T-cells, and nor with memory CD4+ or memory CD8+ T-cell populations (data not shown).

Using an anti-S level $>1000 \mathrm{AU} / \mathrm{mL}$ as the threshold, parameters associated with negative response remain reduced pre-vaccination $\operatorname{lgM}$, IgG3, and CLL therapy within 12 months $(p<0.024,0.046$, and 0.041 respectively), but not for $\operatorname{lgG} 2(p=0.98)$. Interestingly and conversely, the association between reduced pre-vaccination IgA and failure to respond (OR 3.30, $p=0.043$ ) becomes statistically significant, and hence coincides with data reported by Parry et al. ${ }^{20}$ and Herishanu et $a l^{21}$. Both those two reports employed the Roche Elecsys ${ }^{\circledR}$ electrochemiluminescence immunoassay for measuring serologic responses while we used the Abbott Diagnostics assay. Differences in assay performance ${ }^{33,34}$ may contribute to discrepancies between our data and those of Parry et al. ${ }^{20}$ and Herishanu et al. ${ }^{21}$.

CLL and immunocompromised individuals are unable to clear certain viruses such as rhinovirus. ${ }^{11,12}$ For the much more serious COVID-19, long-term shedding of live virus has now been documented in CLL. ${ }^{19,35}$ The failure to develop anti-S antibodies or neutralizing activity is likely a major contributing factor to develop long-term viral replication and shedding. ${ }^{35}$ While the incidence of this issue remains unclear, its occurrence has major 
implications for CLL patients, their families, friends, and the broader community for infection control.

Some CLL patients do respond to vaccination as a recent second report from the Israeli group $^{36}$ demonstrates, and this remains intact for at least 6 months. However, among CLL patients who fail to respond to 2 dose vaccinations, the response rates to third or more doses are likely to be low, and with low levels of anti-S, ${ }^{37}$ and this is consistent with our experience to date. Nevertheless, third and perhaps fourth vaccine doses are the easiest and most immediately available option and supported by our T-cell FluoroSpot data suggesting a functional T-cell anti-COVID-19 response in most patients. These $\mathrm{T}$ cell responses, especially CD8+ T cells, may be important in protection against severe lung disease, and $\mathrm{T}$ and $\mathrm{B}$ cell memory are important in durability of the vaccine response, probably especially in the ageing. ${ }^{38,39}$ Nevertheless, it is clear that there will be a significant group for whom no amount of vaccination will result in seroconversion.

For patients who fail to seroconvert, the ongoing management is likely to remain a challenge for some time and will almost certainly rely on passive immunity. In some settings, hyperimmune COVID-19 immunoglobulin ${ }^{40}$ is available but supply is likely to remain limited. Immunoglobulin replacement therapies may soon have anti-S antibodies detectable, however, the level of antibody and whether it confers any protective activity will be difficult to establish. Furthermore, the antibody levels will reflect vaccination rates in source plasma which is known to be low even in some settings with unrestricted vaccine access. Monoclonal antibody (MAb) preparations recently developed such as sotrovimab ${ }^{41}$ and the antibody combination (REGEN-COV casirivimab and imdevimab) ${ }^{42}$ are currently used in the setting of COVID-19 infection rather than prophylaxis due to the relatively short 
half-life (eg casirivimab and imdevimab, 32 and 27 days, respectively) of these MAb. The very recent data on the high-neutralization MAb cocktail, AZD7442 (co-administered tixagevimab and cilgavimab) ${ }^{43}$ engineered for a prolonged half-life of 9-12 months will make this and similar agents more practical as a prophylactic therapy for those patients who are unable to achieve protective antibodies by vaccination.

In conclusion, the rate of vaccination failure is very high in CLL, especially those on therapy, but also includes many with early-stage disease, and also individuals with MBL, i.e. patients for whom disease-related immune impairment previously had minimal clinical impact. Our data show there is a significant gap between seroconversion with anti-S antibody positivity and the presence of neutralizing antibodies, the latter of which are present in a much smaller proportion of MBL and CLL. It will be important to establish which elements of the immune system best predict Covid-19 protection as many appear to have an intact T-cell response. In any event, the standard precaution measures of masks, hand hygiene, social distancing, and vaccination of close family, friends and contacts will remain important and every CLL patient should remain conscious of these relatively simple precautions. The data from third and more vaccine doses will be important to evaluate as the information becomes available, as will the evaluation of MAbs and other potential passive immune strategies. 
medRxiv preprint doi: https://doi.org/10.1101/2021.10.28.21265549; this version posted October 30, 2021. The copyright holder for this preprint (which was not certified by peer review) is the author/funder, who has granted medRxiv a license to display the preprint in perpetuity.

\section{It is made available under a CC-BY-NC-ND 4.0 International license.}

\section{Acknowledgments}

Robert Traficante, Statistical Revelations Pty Ltd, provided technical support in generating

Figure 3 during preparation of this manuscript.

\section{Authorship Contributions}

YS, JA, JH, KN, AS, PD, IK, LW, NB, SM collected the data. SM, JA, PD, IK confirmed the accuracy of the clinical data. VM, AA, GG, KS, AC, ST performed experiments and interpreted the data. YS and CT compiled the data for statistical analysis. AS performed the statistical analysis. YS and SM prepared the final manuscript, which all authors reviewed and approved.

\section{Conflict-of interest disclosure}

The authors have no conflicts of interest to declare. 


\section{References}

1. Crassini K. R., Zhang E., Balendran S., et al. Humoral immune failure defined by immunoglobulin class and immunoglobulin $\mathrm{G}$ subclass deficiency is associated with shorter treatment-free and overall survival in Chronic Lymphocytic Leukaemia. $\mathrm{Br} J$ Haematol. 2018;181(1):97-101.

2. Freeman J. A., Crassini K. R., Best O. G., et al. Immunoglobulin G subclass deficiency and infection risk in 150 patients with chronic lymphocytic leukemia. Leuk Lymphoma. 2013;54(1):99-104.

3. Forconi Francesco, Moss Paul. Perturbation of the normal immune system in patients with CLL. Blood. 2015;126(5):573-581.

4. Andersen M. A., Eriksen C. T., Brieghel C., et al. and predictors of infection among patients prior to treatment of chronic lymphocytic leukemia: a Danish nationwide cohort study. Haematologica. 2018;103(7):e300-e303.

5. Crassini Kyle R, Best O Giles, Mulligan Stephen P. Immune failure, infection and survival in chronic lymphocytic leukemia. Haematologica. 2018;103(7):e329.

6. Ishdorj G., Beiggi S., Nugent Z., et al. Risk factors for skin cancer and solid tumors in newly diagnosed patients with chronic lymphocytic leukemia and the impact of skin surveillance on survival. Leuk Lymphoma. 2019;60(13):3204-3213.

7. Mulligan S. P., Shumack S., Guminski A. Chronic lymphocytic leukemia, skin and other second cancers. Leuk Lymphoma. 2019;60(13):3104-3106.

8. Falchi L., Vitale C., Keating M. J., et al. Incidence and prognostic impact of other cancers in a population of long-term survivors of chronic lymphocytic leukemia. Ann Oncol. 2016;27(6):1100-1106.

9. Melchardt Thomas, Weiss Lukas, Greil Richard, Egle Alexander. Viral infections and their management in patients with chronic lymphocytic leukemia. Leukemia \& lymphoma. 2013;54(8):1602-1613.

10. Giridhar Karthik V., Shanafelt Tait, Tosh Pritish K., Parikh Sameer A., Call Timothy G. Disseminated herpes zoster in chronic lymphocytic leukemia (CLL) patients treated with B-cell receptor pathway inhibitors. Leukemia \& Lymphoma. 2017;58(8):19731976.

11. Engelmann I., Dewilde A., Lazrek M., et al. In Vivo Persistence of Human Rhinoviruses in Immunosuppressed Patients. PLoS One. 2017;12(2):e0170774.

12. Ohrmalm L., Wong M., Aust C., et al. Viral findings in adult hematological patients with neutropenia. PLoS One. 2012;7(5):e36543.

13. Hu Ben, Guo Hua, Zhou Peng, Shi Zheng-Li. Characteristics of SARS-CoV-2 and COVID19. Nature Reviews Microbiology. 2021;19(3):141-154.

14. Langerbeins Petra, Eichhorst Barbara. Immune Dysfunction in Patients with Chronic Lymphocytic Leukemia and Challenges during COVID-19 Pandemic. Acta haematologica. 2021:1-11.

15. Mato Anthony R., Roeker Lindsey E., Lamanna Nicole, et al. Outcomes of COVID-19 in patients with CLL: a multicenter international experience. Blood. 2020;136(10):11341143.

16. Scarfò Lydia, Chatzikonstantinou Thomas, Rigolin Gian Matteo, et al. COVID-19 severity and mortality in patients with chronic lymphocytic leukemia: a joint study by ERIC, the European Research Initiative on CLL, and CLL Campus. Leukemia. 2020;34(9):2354-2363. 
17. Roeker Lindsey Elizabeth, Eyre Toby Andrew, Thompson Meghan C., et al. COVID-19 in patients with CLL: improved survival outcomes and update on management strategies. Blood. 2021.

18. Goubet Anne-Gaëlle, Dubuisson Agathe, Geraud Arthur, et al. Prolonged SARS-CoV-2 RNA virus shedding and lymphopenia are hallmarks of COVID-19 in cancer patients with poor prognosis. Cell Death \& Differentiation. 2021.

19. Avanzato Victoria A., Matson M. Jeremiah, Seifert Stephanie N., et al. Case Study: Prolonged Infectious SARS-CoV-2 Shedding from an Asymptomatic Immunocompromised Individual with Cancer. Cell. 2020;183(7):1901-1912.e1909.

20. Parry H., Mcllroy G., Bruton R., et al. Antibody responses after first and second Covid-19 vaccination in patients with chronic lymphocytic leukaemia. Blood Cancer Journal. 2021;11(7):136.

21. Herishanu Y., Avivi I., Aharon A., et al. Efficacy of the BNT162b2 mRNA COVID-19 vaccine in patients with chronic lymphocytic leukemia. Blood. 2021;137(23):31653173.

22. Roeker Lindsey E., Knorr David A., Pessin Melissa S., et al. Anti-SARS-CoV-2 antibody response in patients with chronic lymphocytic leukemia. Leukemia. 2020;34(11):3047-3049.

23. Hallek M., Cheson B. D., Catovsky D., et al. iwCLL guidelines for diagnosis, indications for treatment, response assessment, and supportive management of CLL. Blood. 2018;131(25):2745-2760.

24. McCaughan Georgia, Di Ciaccio Pietro, Ananda-Rajah Michelle, et al. COVID-19 vaccination in haematology patients: an Australian and New Zealand consensus position statement. Internal Medicine Journal. 2021;51(5):763-768.

25. Di Ciaccio Pietro, McCaughan Georgia, Trotman Judith, et al. Australian and New Zealand consensus statement on the management of lymphoma, chronic lymphocytic leukaemia and myeloma during the COVID-19 pandemic. Internal Medicine Journal. 2020;50(6):667-679.

26. Tea F., Ospina Stella A., Aggarwal A., et al. SARS-CoV-2 neutralizing antibodies: Longevity, breadth, and evasion by emerging viral variants. PLoS Med. 2021;18(7):e1003656.

27. Mulligan CS, Thomas ME, Mulligan SP. Monoclonal B-lymphocytosis: demographics, nature and subclassification in 414 community patients. Leukemia \& lymphoma. 2011;52(12):2293-2298.

28. Whitaker Jennifer A, Parikh Sameer A, Shanafelt Tait D, et al. The humoral immune response to high-dose influenza vaccine in persons with monoclonal B-cell lymphocytosis (MBL) and chronic lymphocytic leukemia (CLL). Vaccine. 2021;39(7):1122-1130.

29. Kallolimath Somanath, Sun Lin, Palt Roman, et al. Highly active engineered IgG3 antibodies against SARS-CoV-2. Proceedings of the National Academy of Sciences. 2021;118(42):e2107249118.

30. Woyach Jennifer, Tedeschi Alessandra, Munir Talha, et al. Using ibrutinib in earlier lines of treatment results in better outcomes for patients with chronic lymphocytic leukemia/small lymphocytic lymphoma. Leukemia \& Lymphoma. 2021:1-5.

31. Liebers N., Speer C., Benning L., et al. Humoral and cellular responses after COVID-19 vaccination in anti-CD20 treated lymphoma patients. Blood. 2021. 
32. Blixt Lisa, Bogdanovic Gordana, Buggert Marcus, et al. Covid-19 in patients with chronic lymphocytic leukemia: clinical outcome and B-and T-cell immunity during 13 months in consecutive patients. Leukemia. 2021:1-6.

33. Wehrhahn M. C., Brown S. J., Newcombe J. P., et al. An evaluation of 4 commercial assays for the detection of SARS-CoV-2 antibodies in a predominantly mildly symptomatic low prevalence Australian population. J Clin Virol. 2021;138:104797.

34. Narasimhan Madhusudhanan, Mahimainathan Lenin, Araj Ellen, et al. Clinical Evaluation of the Abbott Alinity SARS-CoV-2 Spike-Specific Quantitative IgG and IgM Assays among Infected, Recovered, and Vaccinated Groups. Journal of Clinical Microbiology. 2021;59(7):e00388-00321.

35. Ye X., Xiao X., Li B., et al. Low Humoral Immune Response and Ineffective Clearance of SARS-Cov-2 in a COVID-19 Patient With CLL During a 69-Day Follow-Up. Front Oncol. 2020;10:1272.

36. Herishanu Yair, Avivi Irit, Levi Shai, et al. Six Months Antibody Persistence after BNT162b2 mRNA COVID-19 Vaccination in Patients with Chronic Lymphocytic Leukemia. Blood advances. 2021.

37. Marlet Julien, Gatault Philippe, Maakaroun Zoha, et al. Antibody Responses after a Third Dose of COVID-19 Vaccine in Kidney Transplant Recipients and Patients Treated for Chronic Lymphocytic Leukemia. Vaccines. 2021;9(10):1055.

38. Chen Zeyu, Wherry E John. T cell responses in patients with COVID-19. Nature Reviews Immunology. 2020;20(9):529-536.

39. Cunningham Anthony L, McIntyre Peter, Subbarao Kanta, Booy Robert, Levin Myron J. Vaccines for older adults. bmj. 2021;372.

40. Parikh Devang, Chaturvedi Alok, Shah Naman, Patel Piyush, Patel Ronak, Ray Suma. Safety and efficacy of COVID-19 hyperimmune globulin (HIG) solution in the treatment of active COVID-19 infection-Findings from a Prospective, Randomized, Controlled, Multi-Centric Trial. medRxiv. 2021.

41. Gupta Anil, Gonzalez-Rojas Yaneicy, Juarez Erick, et al. Early Covid-19 Treatment With SARS-CoV-2 Neutralizing Antibody Sotrovimab. medRxiv. 2021.

42. O'Brien Meagan P, Forleo-Neto Eduardo, Musser Bret J, et al. Subcutaneous REGENCOV antibody combination to prevent Covid-19. New England Journal of Medicine. 2021;385(13):1184-1195.

43. Dong Jinhui, Zost Seth J, Greaney Allison J, et al. Genetic and structural basis for SARS-CoV-2 variant neutralization by a two-antibody cocktail. Nature Microbiology. 2021:1-12. 
Tables and Figure legends

Table 1. Patient characteristics

Table 2. IgG anti-spike protein response (negative or positive [i.e. anti-S level>50]) rates in CLL and MBL.

Table 3. Anti-SARS-Cov2 Spike antibody IgG level stratification in CLL, MBL patients and healthy donors post vaccination. Only patients with quantitative anti-spike antibody level available was included in this table.

Table 4. Multivariate analysis for serologic response in CLL patients.

Figure 1. IgG anti-spike antibody levels post vaccination. (A) Changes in IgG anti-S levels pre-vaccination, post D1 and D2. Donors and patients with anti-S values $<1$ (including zero) is shown at $<=1$ on the graph. Values at the top of the graph at D1 and D2 display $n / N$, where $n$ is the number of donors/patients with an anti-S value above the threshold, and $\mathrm{N}$ is the total number of donors/patients with a non-missing value. Thresholds are shown for $>=50$ and $>=1000$. (B) Post D2 IgG anti-spike antibody values. Red bars represent median titer value for each group. 
Figure 2. Odds ratio (OR) for no response to COVID-19 vaccine. (A) Anti-S responses after 2 vaccine doses by age, gender, duration of CLL, IgA, IgM, therapy within last 12 months, ongoing therapy, off therapy in CR/PR, off therapy in relapse, and whether patient was on immunoglobulin replacement therapy, previously on, or both. (B) Anti-S responses after 2 vaccine doses by total IgG and IgG subclass, excluding patients on immunoglobulin replacement therapy. IgG anti-S response (negative or positive [i.e. anti-S value>50]).

Figure 3. Post D2 vaccination neutralizing antibody and T-CLL function assays. (A) $50 \%$ neutralization against the D614G strain and the Delta variant of the SARS-CoV-2 after the second vaccination. Positive and negative results from neutralization assay were split into three stratifications of anti-S responses. (B) CLL patients mount functional T-cell responses to SARS-CoV-2 S protein after vaccination. Frequency of IFN $Y$ and IL-2 producing T cells in response to an overlapping peptide pool covering the complete $S$ protein in a dual color FluoroSpot assay. Each dot represents an individual participant. The red line denotes the median and the dotted line is the threshold for a positive response. SFU= spot forming units. $\mathrm{n}=32$. (C) Image of FluoroSpot show duplicated results of patient 7 and 8 . Green spots are IFNY, and yellow spots are IL-2. Negative control lacked peptides and positive control contained Phytohemagglutinin (PHA). 
Table 1. Patient characteristics

\begin{tabular}{|c|c|c|}
\hline Total number of patients $(n=181)$ & $\operatorname{CLL}(n=160)$ & $\operatorname{MBL}(n=21)$ \\
\hline Males & $56.3 \%(90)$ & $38.1 \%(8)$ \\
\hline Median age (years) & $71.5(22-94)$ & $71(50-86)$ \\
\hline Median age at diagnosis (years) & $60(18-85)$ & $68(48-84)$ \\
\hline \multicolumn{3}{|l|}{ Vaccine } \\
\hline AstraZeneca & $136(85.0 \%)$ & $13(61.9 \%)$ \\
\hline Pfizer & $19(11.9 \%)$ & $4(19.0 \%)$ \\
\hline Moderna & $1(0.6 \%)$ & 0 \\
\hline Unknown & $4(2.5 \%)$ & $4(17.4 \%)$ \\
\hline \multicolumn{3}{|l|}{ Cytogenetics } \\
\hline Del $13 q$ & $79 / 122(64.8 \%)$ & $3 / 5(60.0 \%)$ \\
\hline Del 11q & $14 / 122(11.5 \%)$ & $0 / 5$ \\
\hline Del $17 p$ & $9 / 122(7.4 \%)$ & $0 / 5$ \\
\hline Trisomy 12 & $17 / 122(13.9 \%)$ & $1 / 5(20.0 \%)$ \\
\hline Unknown & 38 & 16 \\
\hline \multicolumn{3}{|l|}{ IGVH } \\
\hline Mutated & 24 & - \\
\hline Unmutated & 6 & - \\
\hline Unknown & 130 & 21 \\
\hline \multicolumn{3}{|l|}{ Treatment status at vaccination } \\
\hline Treatment naïve & $82(51.3 \%)$ & $21(100 \%)$ \\
\hline On-therapy & $32(20.0 \%)$ & 0 \\
\hline Off-therapy in remission (CR or PR) & $40(25.0 \%)$ & 0 \\
\hline Off-therapy in relapse & $6(3.8 \%)$ & 0 \\
\hline \multicolumn{3}{|l|}{ Ig replacement therapy } \\
\hline Currently on & $24(15.0 \%)$ & 0 \\
\hline Prior but not current & $9(5.6 \%)$ & 0 \\
\hline Never on Ig replacement therapy & $122(76.3 \%)$ & $21(100 \%)$ \\
\hline Unknown & $5(3.1 \%)$ & - \\
\hline \multicolumn{3}{|c|}{ Anti-CD20 inhibitor in the last 12 months } \\
\hline Yes & $9(5.6 \%)$ & 0 \\
\hline No & $151(94.4 \%)$ & 0 \\
\hline
\end{tabular}


medRxiv preprint doi: https://doi.org/10.1101/2021.10.28.21265549; this version posted October 30, 2021. The copyright holder for this preprint (which was not certified by peer review) is the author/funder, who has granted medRxiv a license to display the preprint in perpetuity.

It is made available under a CC-BY-NC-ND 4.0 International license.

Table 2. Anti-spike antibody response (qualitative: negative or positive [i.e. anti-S value $>50$ ]) rates in CLL and MBL.

Anti-spike antibody response (negative or positive [i.e. anti-S value $>50$ ]) rates

\begin{tabular}{|c|c|c|c|c|}
\hline Population & Assessment & Response & Frequency/group total & Percentage (Exact $\mathrm{CL}$ ) \\
\hline \multirow{2}{*}{ All CLL and MBL } & \multirow{2}{*}{$\begin{array}{l}\text { Post Dose } 1 \text { IgG spike } \\
\text { protein response }\end{array}$} & Negative & $104 / 135$ & $77.04 \%(69.02 \%, 83.83 \%)$ \\
\hline & & Positive & $31 / 135$ & \\
\hline \multirow{2}{*}{ CLL } & \multirow{2}{*}{$\begin{array}{l}\text { Post Dose } 1 \text { IgG spike } \\
\text { protein response }\end{array}$} & Negative & $99 / 125$ & $79.20 \%(71.03 \%, 85.94 \%)$ \\
\hline & & Positive & $26 / 125$ & \\
\hline \multirow{2}{*}{ MBL } & \multirow{2}{*}{$\begin{array}{l}\text { Post Dose } 1 \text { IgG spike } \\
\text { protein response }\end{array}$} & Negative & $5 / 10$ & $50.00 \%(18.71 \%, 81.29 \%)$ \\
\hline & & Positive & $5 / 10$ & \\
\hline \multirow{2}{*}{ All CLL and MBL } & \multirow{2}{*}{$\begin{array}{l}\text { Post Dose } 2 \text { IgG spike } \\
\text { protein response }\end{array}$} & Negative & $74 / 181$ & $40.88 \%(33.65 \%, 48.42)$ \\
\hline & & Positive & $107 / 181$ & \\
\hline \multirow{2}{*}{ CLL } & \multirow{2}{*}{$\begin{array}{l}\text { Post Dose } 2 \text { IgG spike } \\
\text { protein response }\end{array}$} & Negative & $72 / 160$ & $45.00 \%(37.14 \%, 53.05 \%)$ \\
\hline & & Positive & $88 / 160$ & \\
\hline \multirow{2}{*}{ MBL } & \multirow{2}{*}{$\begin{array}{l}\text { Post Dose } 2 \text { IgG spike } \\
\text { protein response }\end{array}$} & Negative & $2 / 21$ & $9.52 \%(1.17 \%, 30.38 \%)$ \\
\hline & & Positive & $19 / 21$ & \\
\hline
\end{tabular}


medRxiv preprint doi: https://doi.org/10.1101/2021.10.28.21265549; this version posted October 30, 2021. The copyright holder for this preprint (which was not certified by peer review) is the author/funder, who has granted medRxiv a license to display the preprint in perpetuity. It is made available under a CC-BY-NC-ND 4.0 International license.

Table 3. Anti-SARS-Cov2 Spike antibody level stratification in CLL, MBL patients and healthy donors post vaccination. Only patients with quantitative anti-spike antibody level available was included in this table.

\begin{tabular}{|c|c|c|c|c|c|c|}
\hline \multirow[b]{2}{*}{ Anti-spike antibody levels } & \multicolumn{2}{|l|}{ CLL } & \multicolumn{2}{|l|}{ MBL } & \multicolumn{2}{|c|}{ Healthy Donor } \\
\hline & Post D1 & Post D2 & Post D1 & Post D2 & Post D1 & Post D2 \\
\hline$<50$ (negative) & $79.1 \%$ & $42.6 \%$ & $50.0 \%$ & $6.7 \%$ & $0.0 \%$ & $0.0 \%$ \\
\hline 50-249 ('weak') & $14.8 \%$ & $14.2 \%$ & $50.0 \%$ & $26.7 \%$ & $4.3 \%$ & $8.0 \%$ \\
\hline 250-999 ('moderate') & $2.6 \%$ & $16.2 \%$ & $0.0 \%$ & $20.0 \%$ & $21.7 \%$ & $4.0 \%$ \\
\hline 1000-4999 ('strong') & $2.6 \%$ & $17.6 \%$ & $0.0 \%$ & $40.0 \%$ & $65.2 \%$ & $4.0 \%$ \\
\hline 5000-9999 ('high') & $0.0 \%$ & $3.4 \%$ & $0.0 \%$ & $0.0 \%$ & $8.7 \%$ & $36.0 \%$ \\
\hline >10000 ('very high') & $0.9 \%$ & $6.1 \%$ & $0.0 \%$ & $6.7 \%$ & $0.0 \%$ & $48.0 \%$ \\
\hline
\end{tabular}


medRxiv preprint doi: https://doi.org/10.1101/2021.10.28.21265549; this version posted October 30, 2021. The copyright holder for this preprint (which was not certified by peer review) is the author/funder, who has granted medRxiv a license to display the preprint in perpetuity.

\section{It is made available under a CC-BY-NC-ND 4.0 International license .}

Table 4. Multivariate analysis for serologic response in CLL patients.

\begin{tabular}{|l|llcc|}
\hline Population & Effect & Adjusted OR & 95\% CL & p-value \\
\hline All CLL patients & $\begin{array}{l}\text { Pre-vaccination IgM } \\
\text { Reduced vs Normal/high }\end{array}$ & 9.310 & $2.871-30.195$ & 0.0002 \\
\cline { 2 - 5 } & $\begin{array}{l}\text { On treatment vs Treatment } \\
\text { naive/not on treatment }\end{array}$ & 13.091 & $3.420-50.104$ & 0.0002 \\
\hline CLL no IgG treatment & $\begin{array}{l}\text { Pre-vaccination IgG2 } \\
\text { Reduced vs Normal/high }\end{array}$ & 1.190 & $0.512-2.767$ & 0.6858 \\
\cline { 2 - 5 } & $\begin{array}{l}\text { Pre-vaccination IgG3 } \\
\text { Reduced vs Normal/high }\end{array}$ & 2.105 & $0.802-5.530$ & 0.1308 \\
\hline
\end{tabular}


A

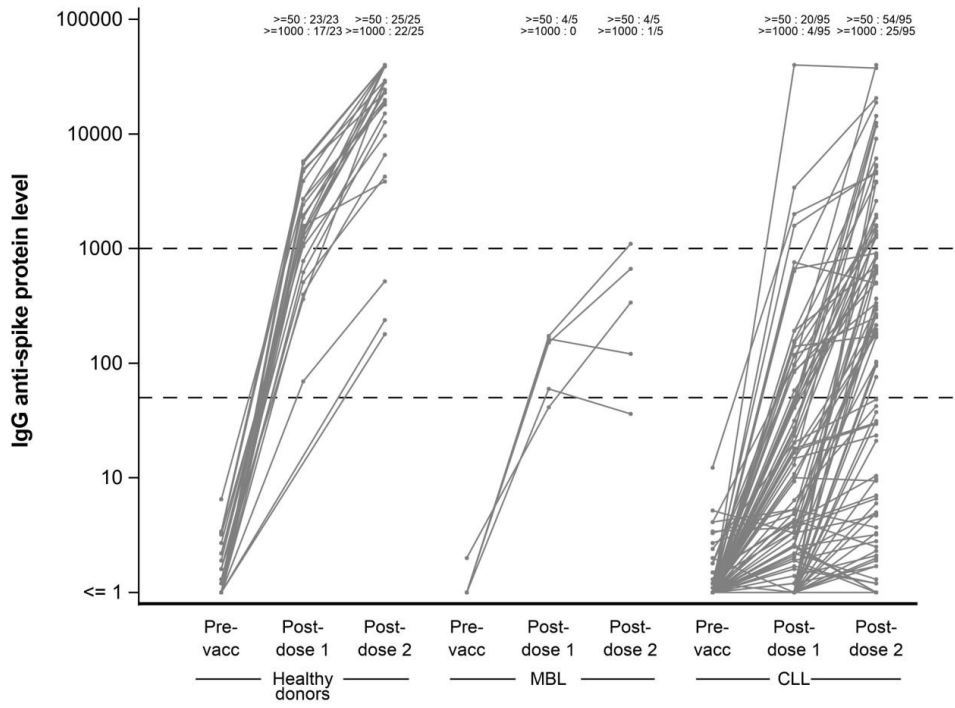

B

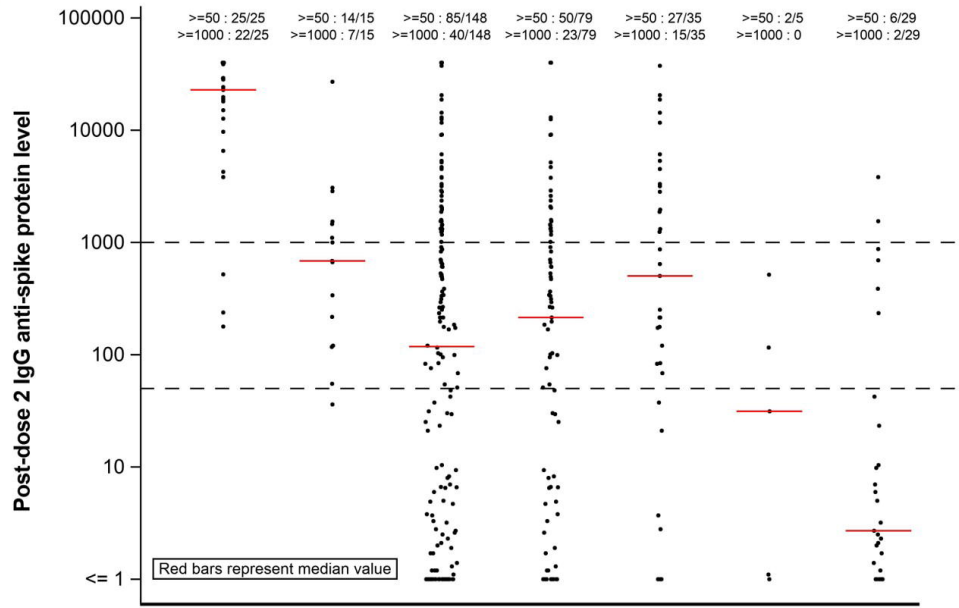

Healthy donors
MBL

CLL
Off-therapy Off-therapy On-therapy remission relapse 


\section{A}

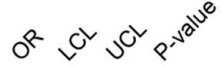

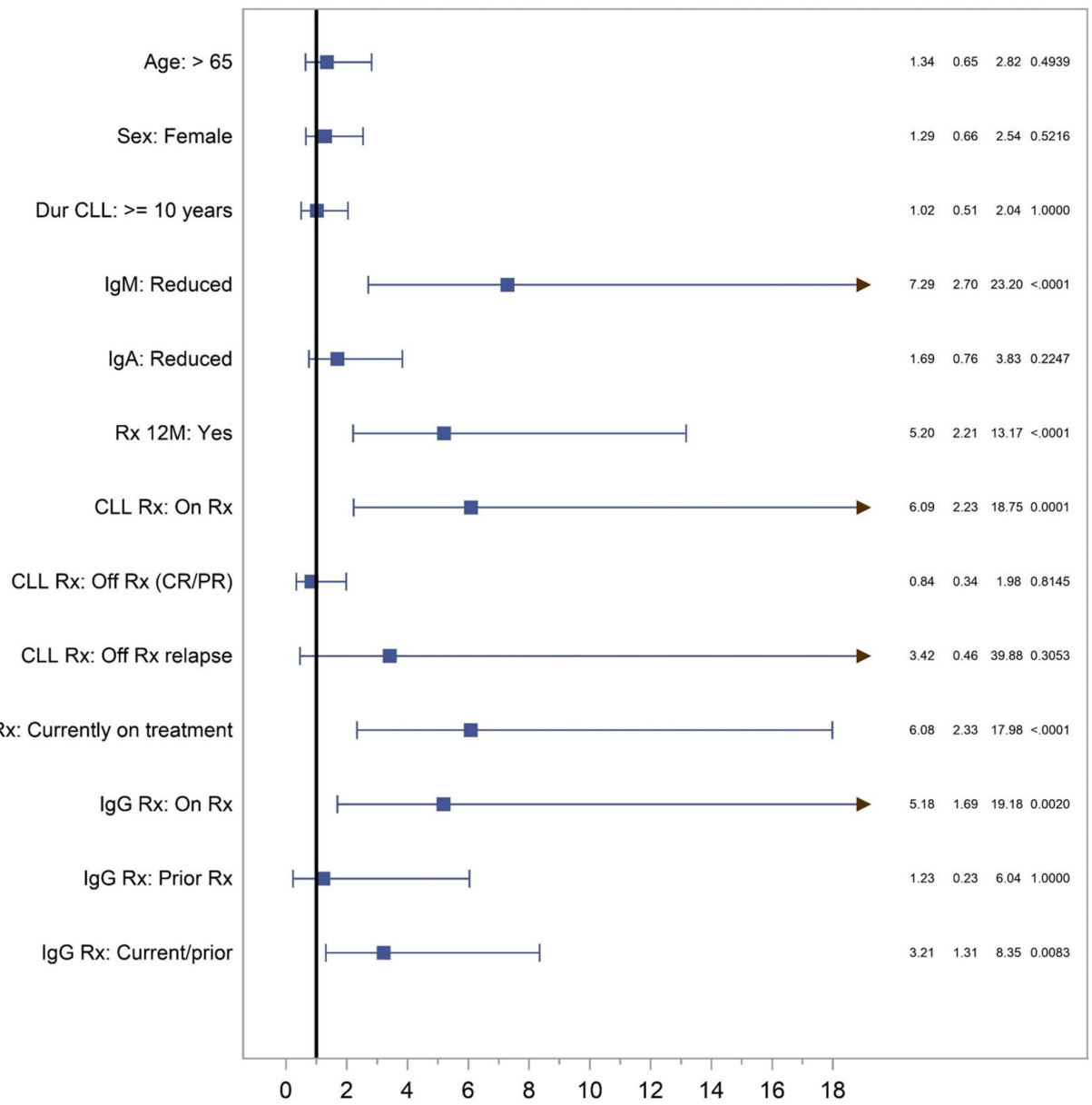

B

Total IgG: Reduced

gG1: Reduced

IgG2: Reduced

Igine Reduced

IgG3: Reduced

IgG4: Reduced

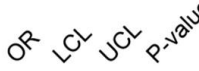

$\begin{array}{llll}1.84 & 0.80 & 4.26 & 0.1647\end{array}$

$\begin{array}{lllll}0.65 & 0.28 & 1.49 & 0.3582\end{array}$

$\begin{array}{lllll}2.52 & 1.06 & 6.23 & 0.0353\end{array}$

$\begin{array}{llll}268 & 1.02 \quad 733 & 0.0458\end{array}$

$\begin{array}{llll}1.56 & 0.52 & 4.68 & 0.5046\end{array}$

Figure 2. Odds ratio (OR) for no response to COVID-19 vaccine. 


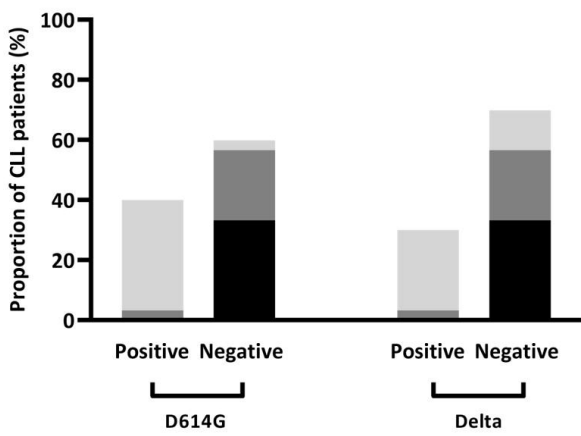

seroconversion $\left[\begin{array}{ll} & \text { High (anti-S protein level }>1000 \mathrm{AU} / \mathrm{mL} \text { ) } \\ \text { Weak/Moderate }(50<\text { anti-S protein level }<1000 \mathrm{AU} / \mathrm{mL} \text { ) } \\ \text { Negative (anti-S protein level }<50 \mathrm{AU} / \mathrm{mL} \text { ) }\end{array}\right.$

B

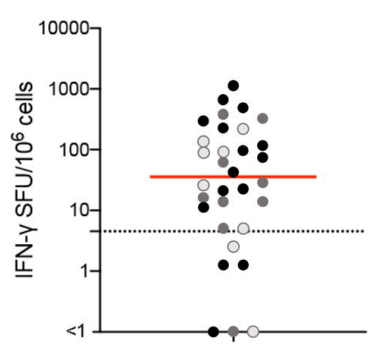

IL-2

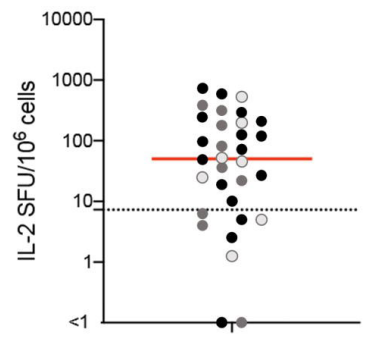

IFN-Y \& IL-2

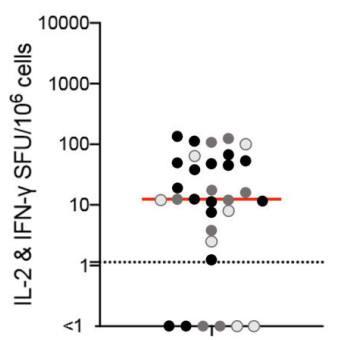

seroconversion $\left[\begin{array}{ll}0 & \text { High (anti-S protein level }>1000 \mathrm{AU} / \mathrm{mL}) \\ \bullet & \text { Weak/Moderate }(50<\text { anti-S protein level<1000 AU/mL) } \\ \bullet & \text { Negative (anti-S protein level }<50 \mathrm{AU} / \mathrm{mL})\end{array}\right.$

C
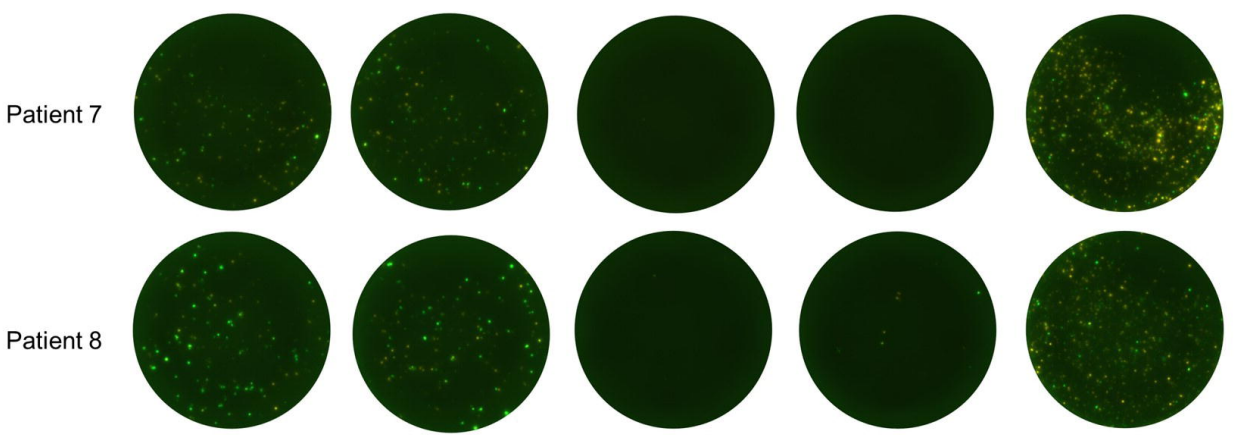

Figure 3. Post D2 vaccination neutralizing antibody and T-CLL function assays. 